\title{
Road Safety: Road Degradation Survey Through Images by UAV
}

\author{
VINCENZO BARRILE ${ }^{1}$, ERNESTO BERNARDO ${ }^{1 *}$, ANTONINO FOTIA ${ }^{1}$, GABRIELE \\ CANDELA $^{1}$, GIULIANA BILOTTA ${ }^{2}$ \\ ${ }^{1}$ Geomatics Lab, Department of civil engineering, energy, environment and materials (DICEAM) \\ Mediterranea University of Reggio Calabria \\ Via Graziella Feo di Vito - 89124, Reggio Calabria
}

\author{
ITALY \\ ${ }^{2}$ Dept. of Planning \\ University IUAV of Venice \\ Santa Croce 191 - 30135, Venice \\ ITALY
}

\begin{abstract}
Preventive safety analyses can help to indicate the presence of situations on a road track potentially at risk for circulation. The key objective of safety analysis is to provide a greater level of safety for all road users. In this paper the evaluation of the road degradation through image by UAV (Unmanned Aerial Vehicle) is presented. A commercial UAV allowing real-time control of a given area and the detection of begging in the flooring required maintenance was used. Particular attention was given to the post-processing procedure of images by experimenting with the use of different ad hoc algorithms that involve the combination of various methodologies. The first tests to provide a continuous monitoring system on the infrastructure were also developed during the acquisition of the images. The proposed system automatically allows to acquire data through a fleet of drones that rise in flight from wireless charging bases, transmitting the acquired data to a cloud platform where they are processed, and finally updating the GIS (Cadastre Roads).
\end{abstract}

Key-Words: - UAV, Image classification, GIS, Image analysis, Segmentation

Received: May 1, 2020. Revised: August 14, 2020. Re-revised: September 4, 2020.

Accepted: September 5, 2020. Published: September 18, 2020.

\section{Introduction}

Road pavements, more than any other civil engineering facilities, are subject to deterioration over time due to different type of loads (e.g. continuous traffic) and atmospheric phenomenon; for these reasons it's important to proper design the structures and perform regular maintenance operations. In the road surface inspection, the data acquisition is the first part of the process to perform regular inspections, to detect and map the discomfort and deterioration. Time of mapping operation, for data acquisition are very important to reduce the overall cost of maintenance and repairs.

Until the early 1980 s, information on road surface conditions was collected through "visual inspections", i.e. workers walking along the road and collecting both visual and quantitative data of the pavement surface by investigating cracks. However, this method of investigation was not very reliable, as the results are subjective, often inconsistent and require time and qualified technician to perform the operation. It was also dangerous as it compromises the safety of operators by exposing themselves to vehicle traffic. Later, to speed up the operations, the inspection was carried out with the help of cameras mounted on trucks, but despite the speed of collecting information, the cost to use this method was expensive and with low-precision images (due to external and atmospheric conditions). Therefore, in recent decades, progress has been made in developing methods to automatically collect images and extract reliable and valuable information on the pavement's conditions. Moreover, road maintenance operations need long time, but also create traffic disturbances.

The Geomatics Laboratory of Mediterranea University of Reggio Calabria has developed a first prototype of continuous roadside survey stations based on the use of UAVs.

\section{Materials and Methods}

In this application, a system was used that allows the automation of road monitoring through a fleet of UAVs and the subsequent logical treatment of the data collected. The main goal of this research is to improve the automation of data processing; improve the quality of the precision in the automatic data 
selection and finally transfer the geolocated data to an online Web-Gis platform.

The methodology used is developed through an automatic process divided into 3 phases: Flight plan setting up; Image analysis; Geolocation of data on the online Web-Gis platform.

We have used a fleet of automated drones connected to the cloud (or a local network) that are automatically recharged through special charging stations located in pre-established points.

With the integration of the cloud platform, a real-time data feed is obtained from the drone fleet, this data is subsequently processed by the algorithms for the selection of the images developed by us. The drones used are DJI Mavic 2 Pro (Fig. 1), equipped with omnidirectional vision sensors and infrared sensors. DJI-branded technologies, such as obstacle detection system, intelligent features such as Hyperlapse, Point of Interest, ActiveTrackTM 2.0, TapFly and QuickShots, as well as assisted piloting systems (APAS), allow you to easily create complex shooting.

Mavic 2 Pro is equipped with a fully stabilized threeaxis gimbal camera, with a 1-inch CMOS sensor (developed in collaboration with Hasselblad) to record $4 \mathrm{~K}$ videos and take 20 -megapixel photos.

Mavic 2 Pro uses the latest technologies to increase the stability and quality of the shooting, reducing the angular vibration range to $\pm 0.005^{\circ}$ for Mavic 2 Zoom and $\pm 0.01^{\circ}$ for Mavic 2 Pro.

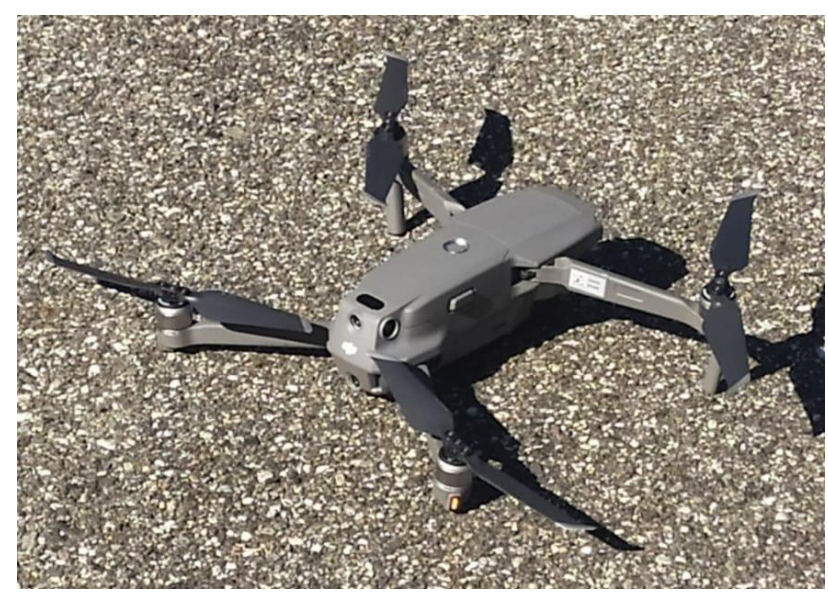

Fig. 1. UAV used for road inspection

The data capture system planned to install two platforms along the path to be detected. These platforms are necessary to allow the charging of the drone battery and the transfer of data necessary for the next processing.

In this research we realized a mini light-weight unattended drone system, including a C500 charging pad, a charging landing gear, a tailored Mavic 2/2 Pro battery, a canopy, an OC (Embedded AI- computer), a LS (local server), an CS (internet server), a T3 (HDMI camera monitoring), a Loudspeaker and a DJI Mavic 2 Pro.

Using this solution, we can control a fleet of automated drones connected to the cloud.

In particular, the process of this research is divided into three phases (Fig. 2):

1. Definition of flight plan, in terms of GSD (Ground Sampling Distance), image overlay and waypoint route: The images are captured following a waypoint path set by the designer. The GSD (Ground Sampling Distance) define the flight height considering the required definition $(\mathrm{cm} / \mathrm{pixel})$; the time to capture images is determined to avoid overlaps and then survey areas that have already been processed

2. Image analysis: pre-elaboration - segmentation classification. In order to improve the quality and precision of the images, the use of different ad hoc algorithms has been experimented involving the combination of various methodologies (Segmentation; Edge detector; Canny filter; Gaussian filter; Support Vector Machine - SVM).

3. Geo-localization of the data on the Web-Gis platform in order to associate each element of the GIS with coordinates relative to the data implemented in the database.
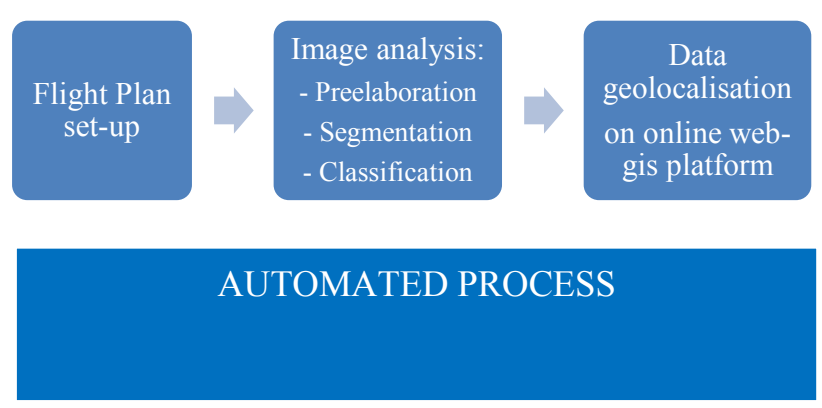

Fig. 2. Workflow for road safety assessment

The operations were tested on the road with little traffic in the territory of the city of Reggio Calabria (RC), via Reggio Campi, in the Tombarello area, on a low density and traffic area (Fig. 3) and the tests were performed at different flight heights in according to the defined flight plan. 


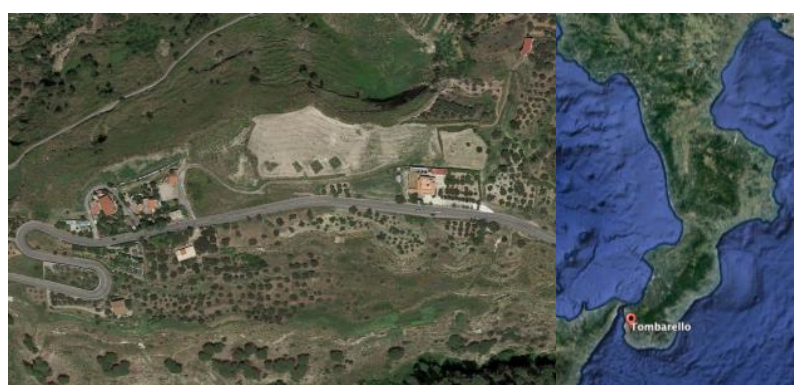

Figure 3. Test area; via Reggio Campi, Tombarello area. Reggio Calabria (RC)

\subsection{Flight plan set-up}

We detected a road with little traffic in the territory of the city of Reggio Calabria and the surveys were carried out in a time with little traffic.

For the acquisition phase of the photographic data set, the DJI Mavic 2 Pro UAV was used. To obtain images with high-quality centimeter accuracy, the image acquisition plan was divided into three phases: definition of the type of plan of image acquisition, definition of Ground Sampling Distance (GSD) and definition of image overlay. The type of image capture was set on the automatic waypoint flight mission for the UAV to perform an automatic flight. The way points have been positioned along the road development. The software automatically calculates the image acquisition plan and the mission settings, has defined the following parameters: flight height (consequently GSD), overlap (\%) and area to be mapped. To obtain centimeter accuracy $(\mathrm{GSD}<1)$ the legal flight height, considering the specifications of the DJI Mavic 2 Pro camera the maximum flight height has been set to 10 meters for vertical flight. Several missions along the way were performed automatically using the Pix $4 d$ flight planning software (Pix4d, Lausanne). To collect photographic information, flight plans were organized with the camera inclination set to $70^{\circ}$. During the flight, the drone has always maintained a constant height with respect to the take-off point, therefore initial tests were necessary to verify that the difference in height could not in any way compromise the safety of flight operations. We also proceeded to verify the absence of any obstacles (electrical cables or subways) along the flight plan. Apps like Flight plan, however, allow us to change the various heights of the way points thus optimizing the procedures necessary for flight.

\subsection{Image analysis}

In order to improve the quality and precision of the images, particular attention was paid to the postprocessing procedure of the images, experimenting with the use of different ad hoc algorithms that involve the combination of various methodologies:
Preelaboration (Sobel and Prewit operator), Segmentation (Edge detector, Canny filter, Gaussian filter) and Classification (SVM). All this, in order to optimize the quality of the image, perform its segmentation and its final classification.

In particular, edge detector is used to mark the points of a digital image where the light intensity changes abruptly. As known, images are the representation of the physical world, sudden changes in the properties of an image are the symptom of important events or changes in the physical world. These changes can be for example: discontinuity of depth, discontinuity of the orientation of the surfaces, modification of the properties of the materials, and variations of the illumination coming from the surrounding environment.

Moreover, in the field of research of image treatment and artificial vision, in particular of the feature extraction branch, the recognition of the contours plays a fundamental role.

The contour recognition operation generates images containing much less information than the original ones, since it eliminates most of the details that are not relevant for the identification of the contours, while retaining the essential information to describe the shape and the structural and geometric characteristics of the objects represented.

In order to improve the quality of the images and the information they contain, multiple algorithms have been used to improve the recognition of the contours of the holes in the image and thus avoid any attribution of deformation of the road to shadows or other noise elements.

The algorithms used were edge detectors, canny and gaussian filters.

Clearly, a Pre-processing has been made previously. Captured images have been converted to grayscale; those with uneven background lighting have been corrected to achieve the same results as evenly lit ones. Preprocessing was based on removing pixels in the image that have an average intensity compared to higher-intensity pixels. In order to improve the image, an attempt was made to eliminate the noise in the image in question through the use of two operators: Sobel and Prewitt (Fig. 4). This procedure is used as early cracks' detection.

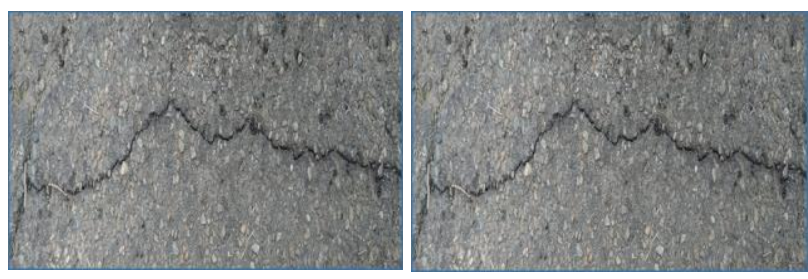

Fig. 4. Preelaboration with Sobel e Prewitt 
Regarding the edge detector, the detection of the edge is achieved by maximizing the signal-to-noise ratio (of the amplitudes) of the gradient. The expression used for the $\mathrm{s} / \mathrm{r}$ ratio is:

$$
s n r=\frac{h_{e}}{\sigma_{n}} S(h), \operatorname{con} S(h)=\frac{\int_{-w}^{0} h(x) d x}{\int_{-w}^{w}[h(x)]^{2} d x}
$$

Where $h_{e}$ is the amplitude of the step while $\sigma_{\mathrm{n}}$ is the standard deviation of the noise.

Points recognized as edge should be as close as possible to the center of the actual edge. For this purpose, a localization factor is defined ( $h$ ' is the derivative of $h$ ):

$$
L O C=\frac{h_{e}}{\sigma_{n}} L(h), \operatorname{con} L(h)=\frac{h^{\prime}(0)}{\int_{-w}^{w}\left[h^{\prime}(x)\right]^{2} d x}
$$

As for the uniqueness of the operator's response, the $\mathrm{x}_{\mathrm{m}}$ distance between two gradient peaks, in the presence of only noise, is supposed to be equal to a $\mathrm{k}$-fraction of the operator's W width:

$$
\mathrm{x}_{\mathrm{m}}=\mathrm{Kw}
$$

The image of response to the operator, when viewed as a 3D surface, is characterized by valleys and reliefs. The maximum curves of the reliefs are called ridges. To get a unique response from the edge detector, we need an algorithm that suppresses all multiple responses, that is, pixels that have high response values to the operator but are not local maximums for it.

The non-maximum suppression is obtained by searching for pixels that are maximum for the magnitude along the direction of the gradient. Only ridges are held by suppressing all other points that are not at the local maximum height.

The quality of the results obtained with the Canny method, higher than that of all other gradient operators, is justified by the fact that the method uses two thresholds, one for the detection of the sharpest edges, the other for the detection of the edges weaker. However, these are only taken into account if they are connected to net.

As for the use of the Cunny method, we can get the image in a grayscale that was smoothed for the first time using a Gaussian filter with a specific standard deviation aimed at reducing noise (example shown in Figure 5), and after that they were gradients for determining the points of the border [1]. We use two threshold values 0.4 (Figure 5.a) and 0.5 (Figure 5.b), with several border points that have been related together. The strong point of the method makes it possible to detect edges even when there is noise and at the same time to detect weak edges. Early margin detection results found that the disage came from the parameters used in the algorithm and that the optimal parameters varied in the wake of each image.

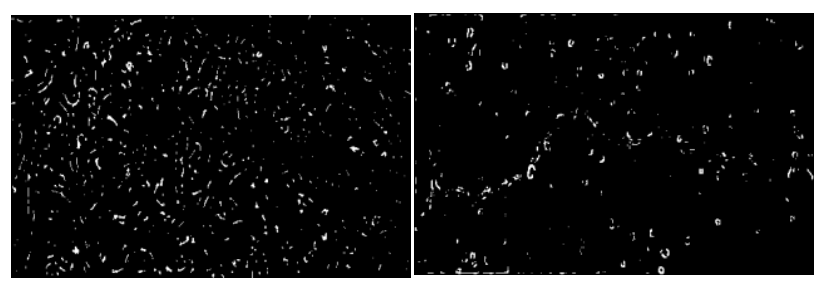

Fig. 5. Processing: a) threshold value $=0.4 \mathrm{~b}$ ) threshold value $=0,5$

In the Segmentation phase, following the cracks detection (Fig. 6.a) an image segmentation tasks were performed: the process was used to partitioning the image into disjointed and homogeneous regions. The purpose of segmentation is to go to simplify and/or change the representation of images to something that is easier to analyze. It is usually used to detect objects and borders (lines, curves, etc.) (Fig. 6.b) but more precisely it is seen as the process by which the pixels of the image are classified that have common characteristics, so each pixel in a region is similar to others in the same region for common characteristics (color, intensity or texture). As the noise in the image increases, the segmentation process becomes more and more complex, and in addition, an uneven light source can make the success of the process more articulated. In this case, the best results were obtained:

- With more sophisticated (nonlinear) algorithms for calculating the size of the gradient (sum of the square of the response of a horizontal edgefinder and the square of the response of a vertical edgefinder);

- With smarter strategies (Canny algorithm) (Fig. 6.c).

Canny's method is an excellent edge detector among the traditional algorithms that are on the market. A border is identified by the relationship between the image and the filter. The filter is chosen on the basis of three effectiveness criteria defined by Canny:

- Good detection capability: The operator has a low probability of not detecting a real edge and a low probability of spotting false edges;

- Good tracking ability: The points highlighted by the operator should be as close as possible to the center of the real edge; 
- Uniqueness of response: operator should provide only one answer at a real edge.

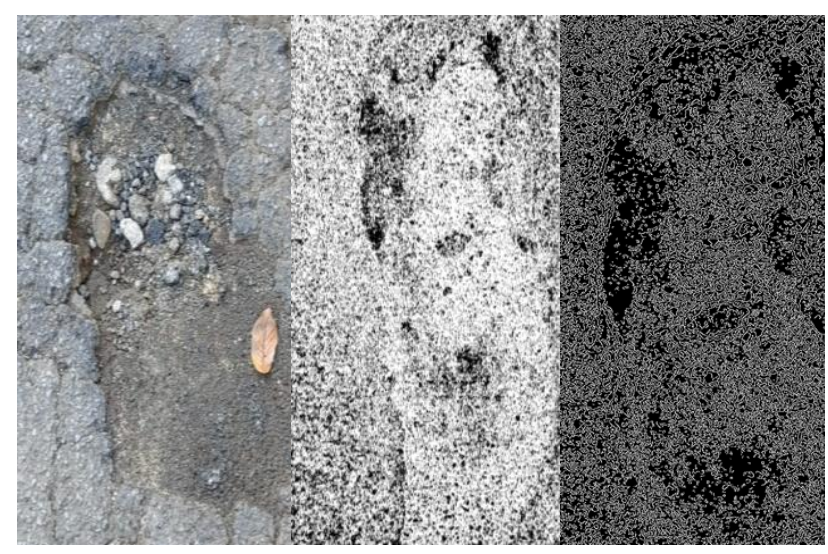

Fig. 6. Preprocessing: a) none; b) edge detector; c) Canny filter

Finally, Classification has been carried out with the SVM method.

In the machine learning sector, support-vector machines (SVM) are supervised learning models with associated learning algorithms that analyze data used for classification and regression analysis.

It is popular in applications such as natural language processing, speech and image recognition and computer vision but achieves maximum effectiveness in binary classification problems. The SVM is based on the idea of finding a hyperplane that best divides a dataset into two classes.

To do this, perform the following steps:

1) Look for a linearly separable hyperplane or a decision limit that separates the values of one class from another. (If there is more than one, look for the one that has the highest margin with the support vectors, to improve the accuracy of the model);

2) If such a hyperplane does not exist, SVM uses a nonlinear mapping to transform the training data into a higher dimension (if we are in two dimensions, it will evaluate the data in 3 dimensions). In this way, the data of two classes can always be separated by a hyperplane, which will be chosen for the division of the data.

Compared with other methods such as artificial neural network, SVMs have significant advantages of high accuracy, elegant mathematical tractability, and direct geometric interpretation [2-9].

We tested four SVMs with different kernels (LIN, HPOL, IPOL, and GRB). In the case of using linear kernel, the KSVM degrades to original linear SVM.
We computed hundreds of simulations in order to estimate the optimal parameters of the kernel functions, such as "the order d" in HPOL and IPOL kernel, and "the scaling factor $\gamma$ " in GRB kernel. The comparison was made through the confusion matrices in which the element of $i^{\text {th }}$ row and $j^{\text {th }}$ column represents the classification accuracy belonging to class $i$ are assigned to class $j$ after the classification $[10 ; 11]$.

In our case, the results showed that the proposed DWT+PCA+KSVM method obtains quite excellent results on both training and validation images. For LIN kernel, the whole classification accuracy was (15 $+137) / 160=95 \%$; for HPOL kernel, was $(17+$ $138) / 160=96.88 \%$; for IPOL kernel, was $(16+$ $141) / 160=98.12 \%$; and for the GRB kernel, was $(18$ $+141) / 160=99.38 \%$. Obviously, the GRB kernel SVM outperformed the other three kernel SVMs.

The comparison results indicate that the used method DWT + PCA + KSVM with GRB kernel performed best, achieving the best classification accuracy as 99.38\%.

Computation time is another important factor to evaluate the classifier.

The time for SVM training was not considered, since the parameters of the SVM keep unchanged after training. We sent all the 160 images into the classifier, recorded corresponding computation time, computed the average value, depicted consumed time of different stages.

For each image, the averaged computation time on feature extraction, feature reduction, and SVM classification is $0.092 \mathrm{~s}, 0.0748 \mathrm{~s}$, and $0.0124 \mathrm{~s}$, respectively. The feature extraction stage is the most time-consuming as $0.092 \mathrm{~s}$. The feature reduction costs $0.0748 \mathrm{~s}$. The SVM classification costs the least time only $0.0124 \mathrm{~s}$. The total computation time for each image is about $0.1792 \mathrm{~s}$, which is rapid enough for a real time analysis.

Referring to the study area previously exposed, the sequence shown in Fig. 7 represents the logical application process referred to in the previous paragraph (further improved step by step thanks to the use of particular local filters and morphological operators) to identify different types of crack that are usually present in common roads (alligator, longitudinal, transverse, non-crack) the cracks. Crack and non-crack regions were extracted from background images using the image processing techniques and methodologies above discussed. The results of this processing are provided in Fig. 7. This figure shows the image for all kind of cracks (alligator, longitudinal, transverse, non-crack), Fig. $7 \mathrm{a}$ input image shows the input images captured by 
the UAV. All images have been resized to $512 \times 512$ pixels to speed up algorithm processing time. The images with non-uniform lighting were adjusted through a morphological operation with a diskshaped filter.

After the application of the above procedures, the results of which are shown in Fig. $7 b$, the images were then processed using both segmentation methodologies (edge detector e Canny) and automatically threshold value. The median filter with a rectangular element of $3 \times 3$ pixels was then applied, and regions with areas greater than 500 pixels were removed. Finally, the structures of the potential crack regions were enhanced through a morphological closure operation with a diamond-shaped filter with a radius of $5 \times 5$ pixels. The final shape of the potential cracks can be seen in the last figure (Fig. 7c). In post processing we proceed to enhance images removing the noise (Fig. 7d) and proceed to detect crack candidates (Fig. 8e) [12 - 19].
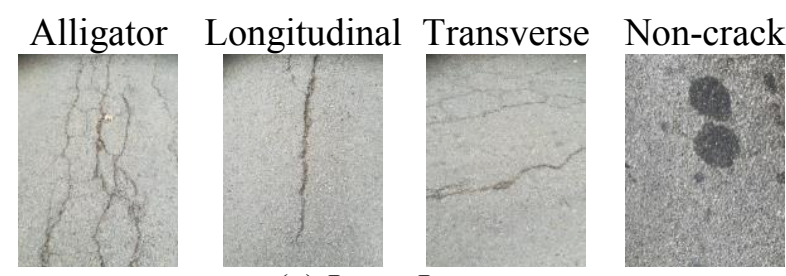

(a) Input Images
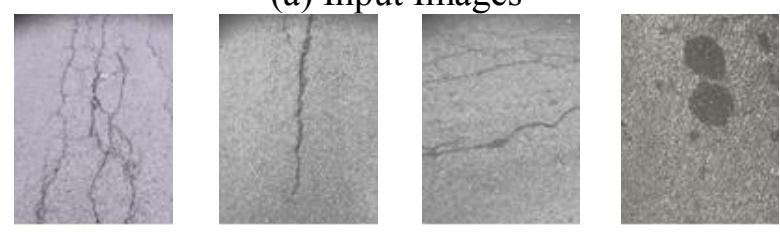

(b) IlluminationCorrection
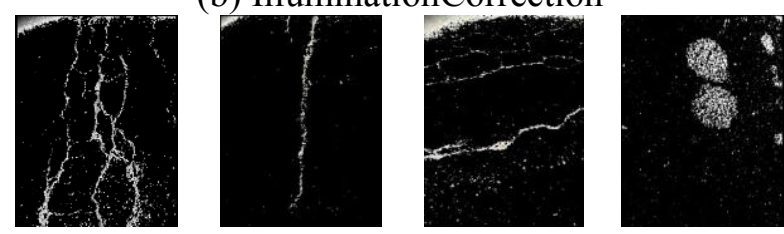

(C) Thresholding based Segmentaion
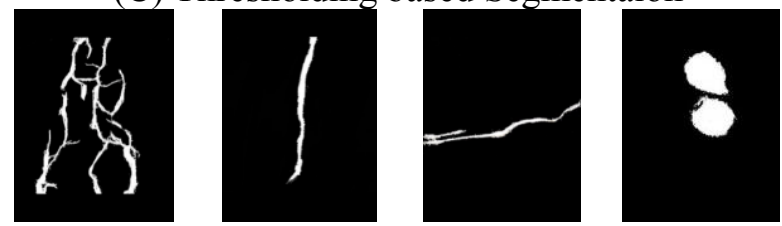

(d) Post Processing and Image Enhancement
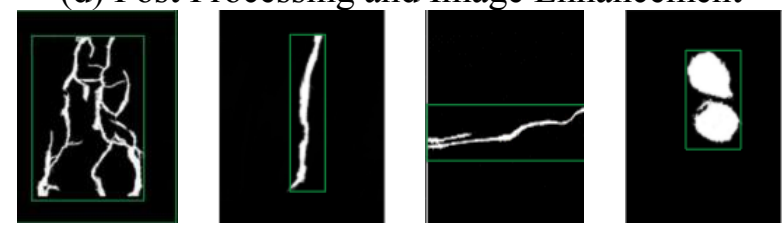

(e) Detected Crack Candidates

Fig.7. Process crack detection
After applying the image analysis techniques to segment and improve the edges and outlines of the images, the crack detection application was carried out through the use of the SVM.

The classification with SVM was carried out in two phases:

1) SVM Training;

2) Performance testing.

In the first phase, the geometric characteristics of the linked components assigned for SVM training were initially calculated. Then, these features were normalized to a range $(0,1)$. Kernel with radial base function (RBF) was chosen as a kernel trick, because the number of instances (connected regions) was not very large and the size of the space transformed with $\mathrm{RBF}$ is infinite. The optimal training parameters for the SVM were found using grid search. During this operation, triple cross-validation was performed to correctly learn the different types of cracks. In this triple cross-validation, the training set was divided into 3 equal subsets. To ensure proper learning, a subset was tested using the trained classifier on the remaining two subsets. The goal was to identify good parameters so that the classifier can predict test data effectively. After learning that the parameters had been determined, SVM was trained with the "One Against All" approach using the MATLAB LIBSVM library.

In the second part, connected regions were tested that were not used during SVM training. The results of the classification have been tabulated in Table 2 .

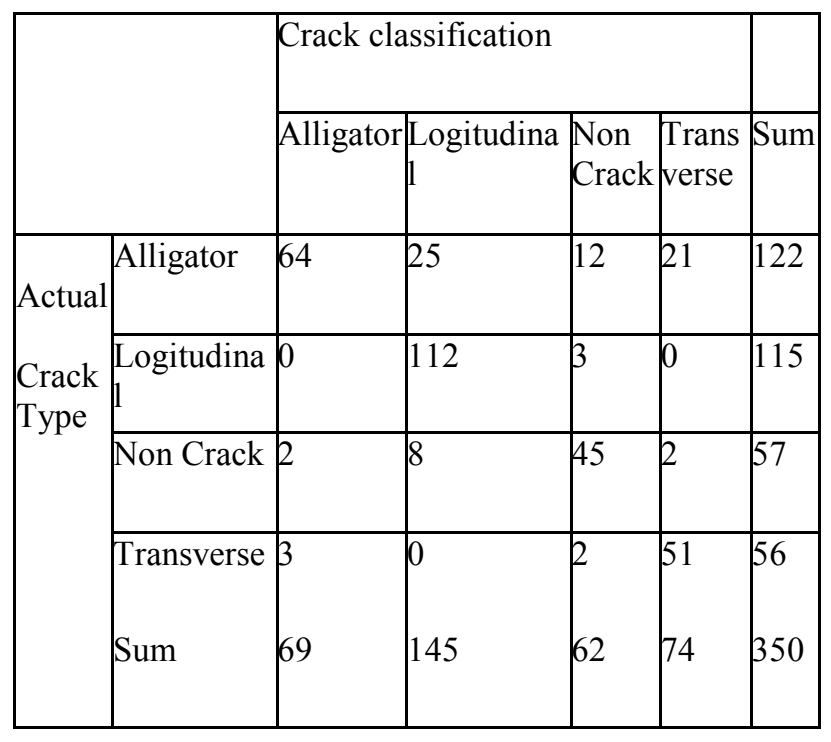

Table 2. Results

In this table, the number "64" indicates that 64 regions of potential crack were correctly classified, meaning there were actually alligator cracks and were considered as such. On the other 
hand, "12" represents the number of alligator cracks that have been misclassified as longitudinal fissures, and so on.

The results of the classification showed that the proposed algorithm has a higher capacity in terms of classification of longitudinal, transverse and noncracked cracks, but the classification of alligator cracks did not have the same because of their complex and variable forms. In fact, some alligatorshaped cracks have been misclassified as other types of cracks, this is mainly due to parts disjointed compared to the rest of the main body of the crack.

Clearly, the high number of the shapes of cracks present in road infrastructure does not always allow to use the proposed procedure and often requires the implementation of neural networks with different layers and many neurons, thus having a considerable "degradation" of the performance of the software to be implemented [20]. Therefore, it was decided to adopt a modular approach: to use multiple neural networks, each of which recognizes not the whole crack but a part of it. This first bottom-up approach is followed by another top-down one, during which, through a deterministic function, the various recognized parts are analyzed, and the object is identified which is unequivocally composed of the parts themselves. The supervised elements are considered a set of "border elements" (cracks) of "color domains" of the border, of "image contained in the border" (internal image). In turn, the "border elements" are considered a composition of predefined "geometric elements"; it is evident that a given "primitive" element, that is not broken down into other elements, can belong to several supervised symbols (those to be recognized).

\subsection{Data Localization Crack GIS (DLCGIS)}

For the crack localization, an automatic procedure called Data Localization GIS (DLCGIS) has been implemented which provides the signaling of the presence of cracks within the GIS using artificial intelligence algorithms, used after a phase of identification of the elements of interest, to automatically store the position of the objects and their alphanumeric data. Then follows the subsequent export of the data acquired within the GIS, where the "historical" update is managed in the existing database, in order to verify the effectiveness of the development of the infrastructure.

Once the various cracks have been identified, they are loaded into a special layer, and "snapped" (moved and anchored) with any polyline present in the layer where the road cartography is represented $[21 ; 22]$.

Specifically, the used procedure works as summarized in the fig. $8,9,10,11,12$.
In more detail, the DLCGIS was developed in four software modules (fig. 8, 9, 10, 11, 12), each with specific functions:

1) The Plug in Module extends the number of objects that can be represented recognizable and classifiable;

2) The kernel, that interact with the users, coordinates the different modules, pre-processing and postprocessing the Input/Output data of the modules themselves;

3) The NNS (Neural Network System) module processes the algorithm implementing the Neural Network trained to recognize the elements of interest;

4) The GIS I/O (Input/Output) Module manages the interface with the GIS software.

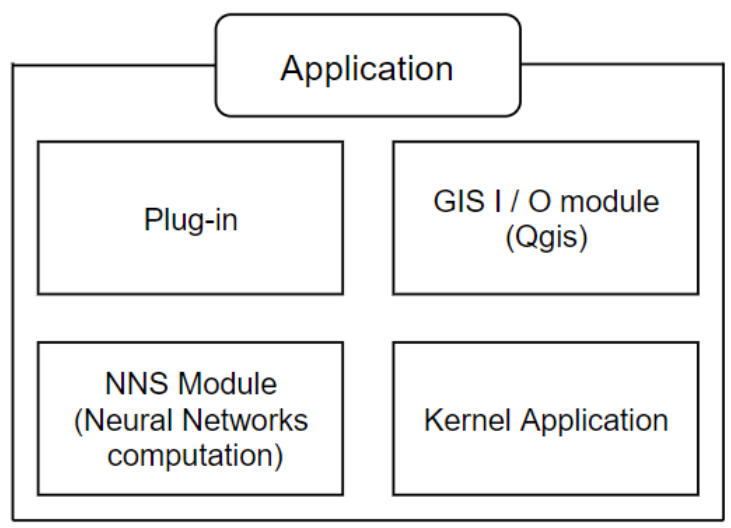

Fig. 8. Schematic rappresentation of the four modules that compose the automatic cracks classification application.

An example of GIS Input/Output module is shown in Fig. 9. In particular, the GIS I/O module is given in Input the files (polylines and polygons) in shp-dbf format (representatives of the road route), returning to Output a space database where the different attributes have been assigned to the objects.

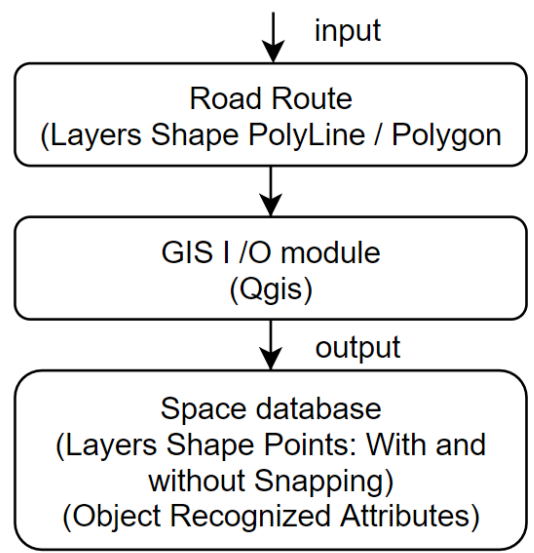


Fig. 9. The GIS I /O module interfaces with the GIS software using files in shp-dbf format.

The NNS (Neural Network System) module instead processes the algorithm implementing the Neural Network trained to recognize the elements of interest; in particular, the image is portioned and processed by different neural networks. The NNS manages the various results of the same in order to recognize and identify the contours of the object being investigated (Fig. 10).

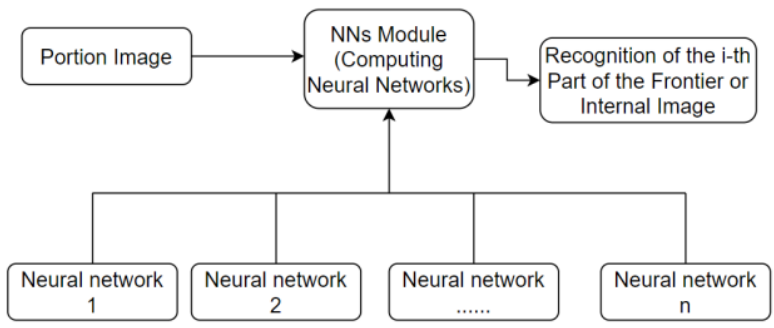

Fig. 10. The NNS Module computes the different models of the Neural Network.

Subsequently, the Plug in Module extends the number of objects that can be represented, recognized and classified, assigning and identifying within the parameter processing range the type of border, the different shades of depth color (Fig.11).

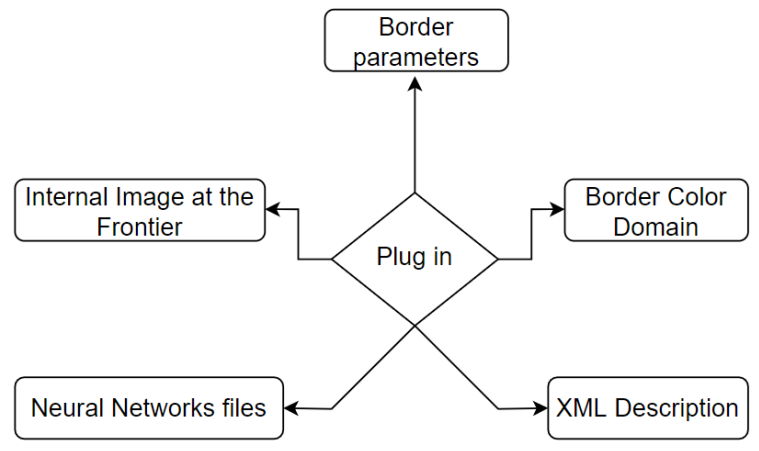

Fig. 11. A Plug-In valid for the application and the parameters that make it up.

The kernel, that interact with the users, coordinates the different modules, pre-processing and postprocessing the Input/Output data of the modules themselves.

The application Kernel works with spatial adjustments (Simple and Intelligent Snapping) on the detected and recognized elements. Through this module the Kernel exports to the GIS one or more specific layers containing the spatial and alphanumeric data (type of signal, etc.) of the identified elements; the data refer to both "adjusted" and unadjusted elements.

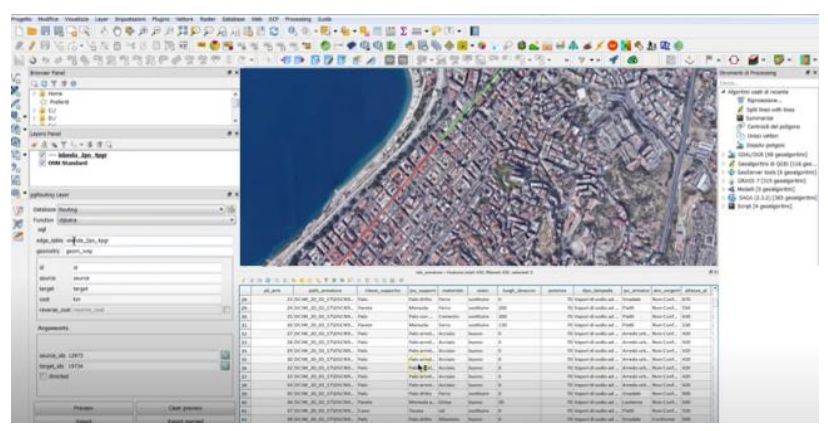

Fig. 12. display on the screen of the properties of the road graph. In red the section with deterioration above $25 \%$.

The system developed for the study area has been tested on additional test areas as shown in fig. 12, and allows, selected a graph, to identify the presence or absence of discrepancies in the roadway (as shown in the figure) with a different color (red) when the roadway affected by the presence of the holes is greater than $25 \%$ of the site area road itself. The generated database can be consulted.

\section{Discussion}

There are different methods and tools for updating the road cadastre, each has its strengths and weaknesses. The solution proposed by us tries to overcome these defects and propose the best solution for updating the road cadastre.

Airborne laser scanning (ALS) is currently widely applied for country-wide DTM generation [23], and corridor mapping (mapping of railroads, streets and highways, and riversides) [24]. ALS is due to transfer costs, multitemporal laser surveys (including change detection, e.g.) are seldom studied and developed.

A summary of ALS can be found in [25] and in [26]. The state-of-the-art in multitemporal ALS research can be found in brief from [27 - 30].

The development of change detection processes is hindered by the lack of good quality data, especially together with hyperspectral images, is expensive due to transfer costs.

Relating the application developed by us with what is reported in the bibliography, the further reduction of costs for the acquisition and management of the data acquired by us is quite evident, being able to reduce the times by $1 / 3$ compared to the alternatives.

Among the alternatives to the UAV for road surface monitoring we can use the mobile mapping system (MMS). 
An MMS consists of the integration of three main hardware components: optical sensors (laser scanner and/or digital cameras), navigation/positioning sensors (IMU/GNSS) and a control and synchronization unit.

Mobile mapping systems using LiDAR technology (also called Mobile Laser Scanning - MLS systems) are the most recent solution. They acquire dense point clouds in real time and offer important advantages, such as: high data acquisition speed, with consequent reduction in costs and time required; high spatial density of the measured data, which allows a dense 3D reconstruction of the detected scene; remote data acquisition, which guarantees the efficiency and safety of the survey $[31 ; 32 ; 33]$.

MMS has multiple advantages, however the processing and management of the data collected and the use of multiple operators often discourages possible users and entails a higher cost than the solution we propose.

In fact, the use of a monitoring solution based on drones allows to automate the monitoring processes eliminating the need to employ more operators, reduces the risk of accidents due to other road vehicles and allows to reach points otherwise inaccessible with an MMS or with other tools.

Furthermore, we carried out a survey (which will be the subject of future papers) on a viaduct both with an MMS and with the UAV system proposed by us. The results obtained verify the effective convenience of times and costs of the system we propose compared to the classic MMS.

Using our proposed and developed system (Fig.13), we automatize both the data acquisition phase, both the data transmission phase and the GIS update phase. We can program and control a fleet of automated drones connected to the cloud (or a local network) that are automatically recharged through special charging stations located in pre-established points. These drones rise in flight and acquired data, that are transmitted in a cloud platform (in this way the drone fleet provides a real-time data feedback, that translates into a faster analysis of the acquired data, providing immediate results compared to conventional methods).

Data processing is done automatically directly in the cloud and finally DLCGIS was created as regards the GIS update phase.

This procedure allows us to reduce time and costs and increase efficiency.

In the future, the next challenge in the field of scientific research to the automation of UAV monitoring will be - the logical treatment of the collected data - improvement of the automation data processing - improvement of precision quality in the automatic selection of data.

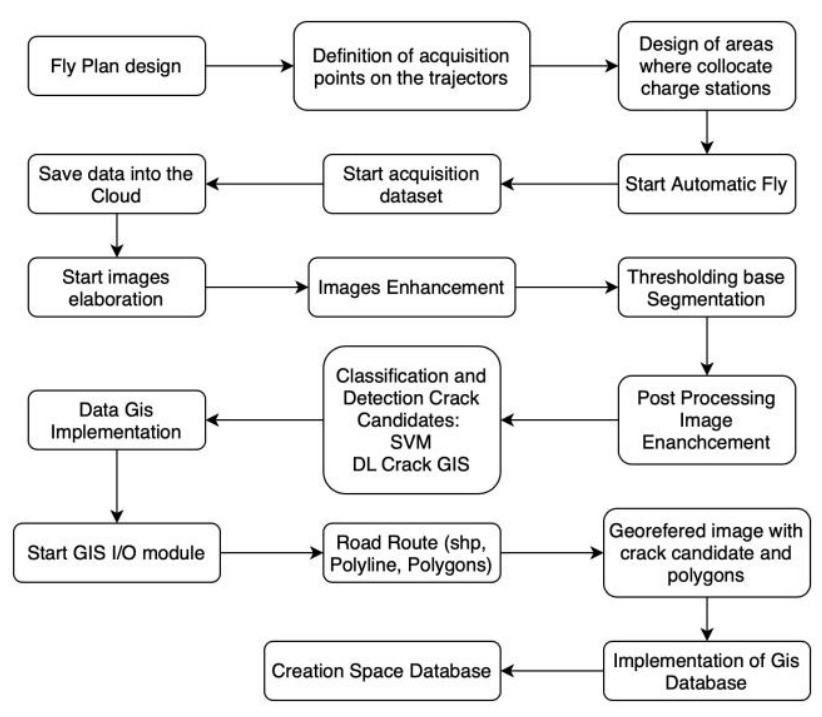

Fig. 13. New methodology flowchart

\section{Conclusions}

The incidence of road pavement degradation is now the cause of numerous accidents. The proposed methodology for the automatic survey can be included for the inspections of road infrastructure and therefore allows who in charge (in the public institution) to be able to plan interventions.

From an economic point of view, the monitoring system proposed by us is economically more advantageous than other solutions that provide similar results; and therefore, if implemented with roadside maintenance functions, this can be an excellent tool for management agencies, which can therefore schedule maintenance based on GIS indications.

The advantages are enormous: economic savings; time saving; greater safety for operators who during the monitoring phase will not have to physically go to the site, thus avoiding being involved in possible road accidents.

The automation of the process allows you to easily identify the areas to be repaired but also to identify over time the areas most subject to wear and tear (areas subject to greater traffic, atmospheric agents, heavy transport, etc.) and therefore improve the programming of the maintenance and resource management.

The Geomatics Laboratory of the Mediterranea University has developed a prototype system for the continuous survey of the state of road paving. The product system, still in progress, sends georeferred dataset to a processing system that through classification algorithms already today 
allows the correct detection of the same dataset with a very satisfying result.

In the future we plan to extend the system, currently limited to the study area only.

Although the National Civil Aviation Authority (ENAC) and the European Union Aviation Safety Agency (EASA) have recently significantly expanded the scope of use of drones, one of the biggest limitations remains the obligation not to fly over crowded areas and obligation to use the operator.

In this regard, we propose to install parachute systems in drones in order to avoid damage to things and people in case of unexpected events.

In addition, it would be advisable to provide, under the drone's trajectory, a buffer zone in order to warn the users that pass through it to be in an area possibly flying over drones.

\section{Reference}

[1] S. Ogawa, K. Matsushima and O. Takahashi, Efficient Pavement Crack Area Classification Using Gaussian Mixture Model Based Features, International Conference on Mechatronics, Robotics and Systems Engineering (MoRSE), Bali, Indonesia, 2019, pp. 75-80.

Doi: 10.1109/MoRSE48060.2019.8998713

[2] T. N. Tonkin and N. G. Midgley, GroundControl Networks for Image Based Surface Reconstruction: An Investigation of Optimum Survey Designs Using UAV Derived Imagery and Structure-from-Motion Photogrammetry, Remote Sens., Vol.8, Issue 9, 2016, pp. 786.

Doi: https://doi.org/10.3390/rs8090786

[3] B. Sekeroglu, K. Tuncal, Image Processing in Unmanned Aerial Vehicles, Al-Turjman F. (eds) Unmanned Aerial Vehicles in Smart Cities. Unmanned System Technologies, Springer, Cham, 2020, pp. 167-179.

Doi: https://doi.org/10.1007/978-3-030-387129_10

[4] F. Dadrasjavan, N. Zarrinpanjeh, A. Ameri, Automatic Crack Detection of Road Pavement Based on Aerial UAV Imagery, Preprints, 2019, 2019070009.

Doi: 10.20944/preprints201907.0009.v1

[5] S. Chen, L. Truong-Hong, D. F. Laefer, E. Mangina, (2018), Automated Bridge Deck Evaluation through UAV Derived Point Cloud, CERI-ITRN2018, Dublin, Ireland, 2018, pp. 735-740.

[6] N. Hoang, Q. Nguyen, A novel method for asphalt pavement crack classification based on image processing and machine learning, Engineering with Computers, Vol. 35, 2019, pp. 487-498.

Doi: https://doi.org/10.1007/s00366-018-06119

[7] Y. Sari, P. B. Prakoso and A. R. Baskara, Road Crack Detection using Support Vector Machine (SVM) and OTSU Algorithm, 6th International Conference on Electric Vehicular Technology (ICEVT), Bali, Indonesia, 2019, pp. 349-354. Doi: 10.1109/ICEVT48285.2019.8993969

[8] A. Ameri, F. Dadrass Javan, N. Zarrinpanjeh, Automatic Pavement Crack Detection Based on Aerial Imagery, Journal of Geomatics Science And Technology 2019, Vol.9, No.1 \#a00641. pp. 145-160.

[9] C. Chen, H. S. Seo, Y. Zhao, B. Chen, J.W. Kim, Y. Choi, and M. Bang, Automatic Pavement Crack Detection Based on Image Recognition, International Conference on Smart Infrastructure and Construction, (ICSIC), 2019, pp 361-369.

Doi: https://doi.org/10.1680/icsic.64669.361

[10] I.L. Al-Qadi, M. Elseifi, and P.J. Yoo, In-situ validation of mechanistic pavement finite element modeling, 2nd Int. Conf. on Accelerated Pavement Testing 1, CD-ROM, 2004.

[11] S. Erlingsson, 3-D FE analyses of HVS tested low volume road structures - comparison with measurements, Proc. 3rd Int. Symp. on 3D Finite Element for Pavement Analysis, Design \& Research, Vol.1, 2002, pp. 339-350.

[12] M.A. Elseifi, I.L. Al-Qadi, and P.J. Yoo, Viscoelastic Modeling and Field Validation of Flexible Pavements, J. Engineering Mechanics, Vol.132, Issue 2, 2006.

Doi: $\quad$ https://doi.org/10.1061/(ASCE)07339399(2006)132:2(172)

[13] H. Yin, S. Stoffels, and M. Solaimanian, Optimization of Asphalt Pavement Modeling based on the Global-Local 3D FEM Approach, Road Materials and Pavement Design, Vol.9, Issue 2, 2008, pp. 345-355.

Doi:https://doi.org/10.1080/14680629.2008.96 90122

[14] M. A. Onyango, Verification of mechanistic prediction models for permanent deformation in asphalt mixes using accelerated pavement testing, PHD Dissertation, Kansas State University, Kansas, 2009.

[15] I. L. Al-Qadi, P.J. Yoo, M.A. Elseifi, and S. Nelson, Creep Behavior of Hot-Mix Asphalt due to Heavy Vehicular Tire Loading, J.of Engineering Mechanics, Vol.135, Issue 11, 2009, pp. 1265-1273. 
Doi: $\quad$ https://doi.org/10.1061/(ASCE)07339399(2009)135:11(1265)

[16] M. Kim, Three-dimensional finite element analysis of flexible pavements considering nonlinear pavement foundation behavior, $P h D$ Thesis, University of Illinois, Urbana, 2007.

[17] C. Huang, R. Abu Al-Rub, E. Masad, and D. Little, Threedimensional simulations of asphalt pavement permanent deformation using a nonlinear viscoelastic and viscoplastic model, $J$. Materials in Civil Engineering, Vol. 23, 2011, pp. 56-68.

[18] L. A. Al-Khateeb, A. Saoud, and M. F. AlMsouti, Rutting Prediction of Flexible Pavements Using Finite Element Modeling, Jordan J. Civil Engineering, Vol.5, No.2, 2011, pp. 173-190.

[19] S. Pandey, K. R. Rao, and D. Tiwari, Effect of geogrid reinforcement on critical responses of bituminous pavements, 25th ARRB Conf., 2012.

[20] Y. Sari, P. B. Prakoso, A. R. Baskara, Application of neural network method for road crack detection, TELKOMNIKA, Telecommunication, Computing, Electronics and Control, Vol.18, No.4, 2020, pp. 19621967.

Doi: 10.12928/TELKOMNIKA.v18i4.14825

[21] V. Barrile, F. Cotroneo, F. Praticò, Automatic updating processes of road surface surveys and surface defects: proposal of an innovative highperformance method, SIIV National Conference Cosenza, Italy, 2006.

[22] V. Barrile, F. Cotroneo, A software for the automatic update of the road cadastre in the GIS environment, Bulletin of the Italian society of photogrammetry and topography, 2006, pp. 923.

[23] G. Sithole, G. Vosselman, Experimental comparison of filter algorithms for bare-earth extraction from airborne laser scanning point clouds, ISPRS Journal of Photogrammetry and Remote Sensing, 2004, Vol.59, Issues 1-2, pp. 85-101.

Doi:https://doi.org/10.1016/j.isprsjprs.2004.05. 004

[24] B. Höfle, M. Vetter, N. Pfeifer, G. Mandlburger, J. Stötter, Water surface mapping from airborne laser scanning using signal intensity and elevation data, Earth Surface Processes and Landforms, 2009, Vol.34, Issue 12, pp. 16351649.

Doi: https://doi.org/10.1002/esp.1853

[25] J. Shan, C. K. Toth, Topographic Laser Ranging and Scanning: Principles and Processing,
Second Edition. CRC Press, Taylor \& Francis Group, 2018.

[26] G. Vosselman, H. G. Maas, Airborne and Terrestrial Laser Scanning, Whittles Publishing, Caithes, GB, 2010, p. 336, ISBN: 978-1904445-87-6.

[27] S. Solberg, E. Naesset, K. Holt Hanssen, E. Christiansen, Mapping defoliation during a severe insect attack on Scots pine using airborne laser scanning, Remote Sensing of Environment, Vol. 102, Issue 3-4, 2006, pp. 364-376.

Doi: https://doi.org/10.1016/j.rse.2006.03.001

[28] T. Vögtle, E. Steinle, Detection and recognition of changes in building geometry derived from multitemporal laserscanning data. International Archives of Photogrammetry, Remote Sensing and Spatial Information Sciences, 35 (Part B2), 2004, pp. 428-433.

[29] X. Yu, J. Hyypp, H. Kaartinen, H. Hyyppä, M. Maltamo, P. Rönnholm, Measuring the growth of individual trees using multitemporal airborne laser scanning point clouds. International Archives of Photogrammetry, Remote Sensing and Spatial Information Sciences, Vol. XXXVI3/W19, WG III/3, III/4, V/3, 2005, pp. 204-208.

[30] X. Yu, J. Hyypp, H. Kaartinen, M. Maltamo, Automatic detection of harvested trees and determination of forest growth using airborne laser scanning. Remote Sensing of Environment, Vol.90, Issue 4, 2004, pp. 451-462.

Doi: https://doi.org/10.1016/j.rse.2004.02.001

[31] V. Barrile, G. Leonardi, A. Fotia, G. Bilotta, G. Ielo, Real-Time Update of the Road Cadastre in GIS Environment from a MMS Rudimentary System, International Symposium on New Metropolitan Perspectives, Springer, Cham, Vol. 101, 2018, pp. 240-247.

Doi: $\quad$ https://doi.org/10.1007/978-3-31992102-0 26

[32] A. Mancini, E. S. Malinverni, E. Frontoni, P. Zingaretti, Road pavement crack automatic detection by MMS images, 21st Mediterranean Conference on Control and Automation, 21st Mediterranean Conference on Control and Automation, Chania, 2013, pp. 1589-1596, Doi: 10.1109/MED.2013.6608934

[33] Z. Florkova, L. Duris, M. Veselovsky, S. Sedivý and D. Kovalova, Three-dimensional mobile mapping system and its use in road engineering, MATEC Web Conf., Vol. 196, No.04082, 2018, Doi:https://doi.org/10.1051/matecconf/2018196 04082

\section{Creative Commons Attribution License 4.0 (Attribution 4.0 International, CC BY 4.0)}

This article is published under the terms of the Creative Commons Attribution License 4.0

https://creativecommons.org/licenses/by/4.0/deed.en_US 